\section{REVISTA}

Actualidades Investigativas en Educación

\title{
CRISIS DE LA REFORMA EDUCATIVA ARGENTINA EN UN CONTEXTO NEOLIBERAL. LOS HANDICAPS DE LA INCLUSIÓN EDUCATIVA Y LABORAL JUVENIL
}

CRISIS OF THE ARGENTINEAN EDUCATIONAL REFORM IN THE NEOLIBERAL CONTEXT. THE HANDICAPS OF THE EDUCATIONAL AND LABORAL INCLUSION OF YOUTH PEOPLE

Volumen 11, Número 1

pp. $1-29$

Este número se publicó el 30 de abril de 2011

Pablo Christian Aparicio

La revista está indexada en los directorios:

LATINDEX, REDALYC, IRESIE, CLASE, DIALNET, DOAJ, E-REVIST@S,

La revista está incluida en los sitios:

REDIE, RINACE, OEI, MAESTROTECA, PREAL, HUASCARAN, CLASCO 


\title{
CRISIS DE LA REFORMA EDUCATIVA ARGENTINA EN UN CONTEXTO NEOLIBERAL. LOS HANDICAPS DE LA INCLUSIÓN EDUCATIVA Y LABORAL JUVENIL
}

CRISIS OF THE ARGENTINEAN EDUCATIONAL REFORM IN THE NEOLIBERAL CONTEXT. THE HANDICAPS OF THE EDUCATIONAL AND LABORAL INCLUSION OF YOUTH PEOPLE

Pablo Christian Aparicio ${ }^{1}$

Resumen: Las reformas educativas realizadas en Argentina durante la década del 90, estuvieron fuertemente condicionadas por la introducción de un nuevo modelo político y social sustentado bajo los cánones del Neoliberalismo, que conllevaron a una redimensión paradigmática del rol del Estado, de la sociedad civil y del mercado. Basados en las conclusiones de tres proyectos de investigación desarrollados en Argentina, el presente artículo se propone analizar las consecuencias de la nueva política educativa que si bien abogó -en sus presupuestos ideológicos y técnicos- por la superación de los déficits organizativos y administrativos y la reducción de las restricciones en el acceso democrático al sistema educativo, en los hechos se observa que muchos problemas pedagógicos no fueron ponderados como prioridades. La falta de articulación entre las ofertas educativas, las demandas en el campo laboral y económico, así como las posibilidades objetivas de participación de los jóvenes, respondieron a una carencia de tipo estructural que encarnara, a posteriori, un hándicap para la implementación efectiva de las nuevas políticas y programas. Con todo, las reformas políticas implementadas han tenido consecuencias regresivas para la consolidación de un sistema educativo competente y democrático. La falta de referentes políticos e institucionales capaces de visibilizar las potencialidades y las demandas de los jóvenes y la planificación de acciones sinérgicas capaces de mejorar la articulación entre las ofertas educativas, el mundo del trabajo y la vida social, explican el por qué de la exclusión y vulnerabilidad todavía vigente en el mundo juvenil argentino.

Palabras clave: EDUCACIÓN, JÓVENES, REFORMAS POLÍTICAS, PARTICIPACIÓN SOCIAL, TRANSICIONES JUVENILES, TRABAJO

\begin{abstract}
The educational reforms carried out in Argentina in the 1990s, were highly influenced by the introduction of the new political and social model of neoliberalism, what led to a new paradigm on the role of the State, the society and the market. Based on the conclusions reached by three research projects carried out in Argentina, this article aims at analysing the consequences of the new educational policies. These advocated - in their ideological and technical framework - for the elimination of previous organisational and administrative deficiencies in the democratization of the education. In reality, many problems of a pedagogical nature were not considered as political and technical priorities. The lack of coordination in the educational offer, the demands that rose in the employment and economical sectors and the participation opportunities of the young population were the result of structural shortcomings. These represent a handicap, a posterior, for the implementation of new policies and programs. All in all, the political reforms have had negative consequences for the consolidation of competent and democratic educational system. The lack of political or institutional referents serving as guides or models capable of referring to the potentials and demands of the young population, the incomplete development of synergistic programs in the educational offer, the employment sector and social interaction, all these explain the current situation of exclusion and vulnerability in the argentine young sector of the population.
\end{abstract}

Key words: EDUCATION, YOUTH, POLITICAL REFORMS, SOCIAL PARTICIPATION, TRANSITION OF YOUTH, WORK

\footnotetext{
${ }^{1}$ Unidad de Investigación del instituto Universitario de Ciencias de la Educación, Universidad de Salamanca (España).
}

Dirección electrónica: pabloaparicio@usal.es

Artículo recibido: 10 de enero, 2011

Aprobado: 25 de abril, 2011 


\section{Introducción}

El presente artículo se basa en las conclusiones derivadas de tres proyectos de investigación, desarrollados en Argentina entre el año 2000 hasta la fecha. El primero de los proyectos dio origen a la tesis doctoral titulada "La política educativa argentina y el incremento de la desocupación juvenil. Orientaciones y estrategias alternativas de integración socio - educativa desde el aporte de las Políticas de Transición y la Pedagogía Social Alemana"2. Los otros dos proyectos forman parte de las actividades del Consejo de Investigación de la Universidad Nacional de Salta: 1) La cultura institucional educativa en una institución de educación superior no - universitaria, tramas de trabajo y tramas de relaciones. Incidencia en la dinámica institucional (Proy. $\mathrm{N}^{*} 1750 / 0$ ); y 2) La construcción de la cultura institucional educativa en contexto social crítico. El entorno de empobrecimiento y sus consecuencias en la dinámica institucional. Estudios de casos (Proy. $\left.\mathrm{N}^{*} 1410 / 0\right)^{3}$.

\section{1) Educación como factor estratégico de desarrollo: ¿decoro retórico o meta pendiente?}

La educación representa en el contexto de la globalización un axioma estratégico, desequilibrante y significativo de producción y reproducción social. Inspirados en su reforma y reconceptualización paradigmática se han depositado, progresivamente, un cúmulo polisémico de exigencias, esperanzas y desafíos en las sociedades de todo el mundo occidental.

La búsqueda de una resignificación adecuada, basada en los prismas de la equidad, democracia, eficiencia y efectividad, han motivado dentro de la comunidad educativa, el sector político local y las entidades de cooperación técnica y financiera internacional la inauguración de reformas orientadas a reformular la política educativa y social.

En el contexto argentino y latinoamericano se coincidió, previa a la instrumentación de las reformas estructurales en el plano político educativo, donde los sistemas educativos y todos sus dispositivos políticos, institucionales, profesionales, científicos, curriculares y tecnológicos dada la imperiosidad de los cambios registrados en el campo económico, laboral, cultural y social a nivel global y local requerían de una revisión crítica sustancial.

\footnotetext{
${ }^{2}$ Tesis presentada y defendida en el Instituto de Ciencias de la Educación de la Eberhad - Karls universität Tübingen, Alemania.

${ }^{3}$ Estos proyectos están dirigidos por el Magíster Sergio Ignacio Carvajal.
} 
Este replanteo sustancial de la propuesta educativa apuntalaba a posibilitar una interpelación de su perfil político, filosófico y epistemológico, su organización curricular, didáctica y práctica, la impronta histórica social de sus procesos de aprendizaje y enseñanza vigentes, y el impacto biográfico y personal de los capitales, disposiciones y conocimientos socializados por las instituciones educativas en todas sus modalidades y niveles.

Vale decir, desde esta reformulación categórica, se suponía sería plausible acceder a una introducción significativa de los cambios requeribles en vistas a confeccionar estrategias de integración social, alternativas superadoras de los déficits prevalecientes al interior del sistema educativo y puentes comunicantes entre el campo educativo y el resto de los campos sociales. He ahí algunos de los argumentos más contundentes que inspiraron y justificaron la consecución en la práctica de una reforma que abogaba por replantear "desde las bases" la política educativa pública y el resto de las herramientas políticas, jurídicas e institucionales que definen la vida histórica de la sociedad.

En este nuevo escenario de transformaciones vertiginosas y determinantes, se produce una suerte de exaltación del poder estratégico y movilizador del saber en lo concerniente a la organización de las sociedades, puesto que en nuestros días educar adecuadamente se ha tornado indudablemente en un mecanismo de vasta importancia para la construcción de sociedades más desarrolladas, competitivas, equitativas e integradas. Pero, del mismo modo, el no acceso y la privación de estos bienes proponen como contra cara de la misma historia, sutiles mecanismos de desplazamiento social, negación cultural, desafiliación política e institucional y desmedramiento real y simbólico.

Es por esta razón que cualquier nueva propuesta de reforma educativa o social no puede concebirse unívocamente como una directriz de cambio social aislado, autoreferencial y sobredeterminante, que por antonomasia, puede infringir mecánica y causalmente los objetivos perseguidos sobre el resto de los ámbitos políticos y sociales. Por lo contrario, esta intencionalidad de cambio -que representan los programas de reforma dentro de cualquier área de la vida social- se circunscribe siempre dentro de una multiplicidad de variables, restricciones, condiciones y potencialidades de tipo histórico, social, cultural, material, económico e ideológico que se comportan de modo complejo, discontinuo y muchas veces imprevisible atraviesan dialécticamente la realidad social.

Esta complejidad aludida fue desatendida por el sector político, burocrático y técnico encargado de transponer la reforma en los espacios de formación educativa, y es 
específicamente en este escenario donde hoy está en juego la formación adecuada de los niños y jóvenes en Argentina.

Por ello, es que la superación de la frustración como herencia de la reforma educativa argentina debería servir de basamento para la orientación de un cambio real y que resulta aún pendiente en nuestros días pese a las inversiones y los cambios acometidos en pos de concretar esta meta.

La búsqueda de estas respuestas se inscribe como un objetivo neurálgico en el presente trabajo; para ello, se intentará brindar nuevas dimensiones de análisis y parámetros de intervención práctica dentro del área de formación de jóvenes expuestos a problemas como la vulnerabilidad, "múltiples pobrezas" ${ }^{4}$ y exclusión social. Con este objetivo se explorarán, seguidamente, algunas de las características anómalas presentes en la política educativa argentina, que obstaculizan en los jóvenes el despliegue de sus potencialidades cognitivas, motivacionales e identitarias y la objetivación histórica de sus derechos, anhelos y proyectos personales biográficos.

\section{2) Una mirada crítica sobre la efectividad y la relevancia de las reformas estructurales}

"No debe permitirse que la tesis de que el mercado está en la
naturaleza humana quede incontestada; a mi juicio, es el ámbito
más crucial de la lucha ideológica de nuestros días". (Jameson, 1996, p. 202)

A través de la implementación de múltiples reformas políticas en el terreno educativo, social, laboral, jurídico, tecnológico y científico se efectivizaron en el marco de la reestructuración del Estado numerosas privatizaciones, desregulaciones, descentralizaciones y concentraciones simultáneas que tendieron a aplicar clausuras provisorias y definitivas de determinadas estructuras y funciones públicas, especialmente, dentro del área de servicio y de producción, acometidas en pos de consagrar la eficiencia y la racionalización del gasto público (Sirvent, 1999).

\footnotetext{
${ }^{4}$ Las Múltiples Pobrezas no se agotan en el diagnóstico de las carencias que hacen a la satisfacción de las necesidades tradicionalmente llamadas básicas u obvias, que ya no son tan obvias, tal como por ejemplo trabajo, vivienda, salud, educación sino que abarca el estudio de una completa realidad de Pobrezas (en plural) en relación con carencias en la satisfacción de necesidades fundamentales, (...) como la necesidad de protección o cuidado, la necesidad de pensamiento reflexivo o de entendimiento y la necesidad de participación política. Cualquier necesidad
} 
Con este criterio de reducción y saneamiento del déficit fiscal y la modernización del aparato estatal en su integralidad -paradójicamente exceptuando las estructuras corruptas, partidistas e improductivas- se sometieron a un proceso de reestructuración que afectó a la mayoría de las instituciones y dependencias públicas en el ámbito de la salud, la seguridad social, la educación, la comunicación, el transporte, la vivienda, el trabajo, etc. La reducción del gasto público, la desafiliación y desautorización del Estado como principal regulador y garante del bienestar social y la paralela y abrupta institucionalización del mercado como nueva lógica de organización social constituyen las modificaciones más significativas sobre los cuales se erige la sociedad de los años 90 (Filmus et al., 2001; Fitoussi y Rosenvallon, 1997).

Todos estos antecedentes dan cuenta de la vigencia de un novedoso e intrincado tejido social al cual asistimos hoy por hoy todos los latinoamericanos; dicho escenario está fuertemente determinado por múltiples contradicciones, inviabilidades y progresivas dinámicas de exclusión, suscitadas por la proyección de los tópicos políticos neoliberales, cuyos resultados han incrementado y legitimado una indiscutible dualización social, fundada en la desigual distribución de los bienes materiales, culturales y sociales.

La brecha social planteada -casi antagónicamente- entre sectores ricos y pobres, con una clase media en paulatino desgranamiento y desagregamiento, se engrosa considerablemente sin poder encontrar en la actualidad una maya de contención o políticas sociales tendientes a la retención y asistencia, capaces de moderar un proceso de "centrifugación social" 5 que se manifiesta en la acelerada y polisémica socialización de la incertidumbre, el atomismo, el miedo y la inseguridad social de sectores, grupos e individuos.

En Argentina, a partir de la introducción de reformas y programas políticos con fuerte arraigo económico y orientación ideológica neoliberal, se ha constatado que dentro del campo político pedagógico -entendido como cuerpo e instancia ejecutiva de organización social de los bienes de la cultura- las prioridades y los objetivos específicos en materia de formación, desarrollo, enseñanza y aprendizaje han tendido, por una parte, a simplificarse tornándose en una mera variable cuantificable dentro del nuevo modelo de gestión de políticas públicas, por

humana que no es adecuadamente satisfecha socialmente revela una pobreza social y genera procesos de exclusión y de aumentos de la violencia internalizada en las relaciones sociales. Izaguirre en Sirvent, 1996b, p. 202

${ }^{5}$ La centrifugación social (como desarticulación del modelo incluyente), la privación de lo político y la preeminencia de la economía divorciada de la ética del bien común constituye rasgos decisivos del escenario argentino de los años 90. La heterogeneidad y la diversidad de la exclusión se expresa en su ataque cruzado a lo largo de toda la estructura social: no todos quedan simultáneamente afuera, ni con la misma severidad. Las combinaciones de exclusión son variadas. (Mancebo, 1998, pp. 193-194) 
otro parte, la aportación de la educación a los procesos de desarrollo en este contexto ha tendido a desdibujarse simultáneamente con la injerencia del Estado sobre asuntos públicos condicionado por el avance de la intervención y protagonismo del mercado y de sus lógicas operativas en torno a lo viable, lo rentable y lo exitoso (Friedman, 1993). Esta transfiguración de la política educativa se condice con la filosofía subyacente en el nuevo paradigma neoliberal que sobrestima y reduce en máximas econométricas de costo y beneficio toda la pluridimensionalidad inherente a los problemas, las exigencias y las necesidades sociales pertenecientes a grupos y a contextos reales, y que en el caso argentino se han derivado en el incremento de los índices de desempleo, subempleo y precarización del trabajador y su trabajo; la expansión progresiva de la pobreza, la vulnerabilidad social y los procesos de exclusión social; el elevado porcentaje de hogares imposibilitados de cubrir sus necesidades básicas, etc. (Tenti Fanfani, 1996)

Indudablemente, la disociación reduccionista y antagónica planteada entre las prioridades del discurso económico y el resto de los discursos sociales (éticos, culturales y políticos) tornan a la realidad en un fenómeno harto complejo, paradojal y ambivalente ${ }^{6}$, desde donde se reproducen las rupturas y las contradicciones sociales en progresiva acentuación (Aparicio, 2004).

Esta tendencia a la reificación de la "lógica del sistema" (Lechner, 1999) consagra el carácter regresivo, dictatorial y ahistórico de los planteamientos neoliberales, que en un contexto de desigualdad, precariedad y extravío generalizado de bases democráticas, solidarias y participativas de la organización social, solo propone la lógica del mercado como alternativa unívoca para la superación y neutralización de las dificultades estructurales de las comunidades. Por esta razón, se afirma que este discurso, dado su reduccionismo, deviene en utópico o en panacea, cuyos beneficios y ventajas en la vida social se aseguran para una escasa minoría en desmedro de una gran mayoría dentro de la estructura social.

Al respecto, comentan Morín y Nair (1998, p. 87)

estamos en un período políticamente regresivo, la política reducida a la economía, y mentalmente regresiva, las ideas gregarias (....) cuando uno evoca la mundialización, el

\footnotetext{
${ }^{6}$ La complejidad no es una receta para conocer lo inesperado (...). La complejidad se sitúa en un punto de partida para una acción más rica, menos mutilante. Yo creo profundamente que cuanto menos mutilante sea un pensamiento, menos mutilará a los humanos. Hay que recordar las ruinas que las visiones simplificantes han producido (y producen) no solo en el mundo intelectual sino en la vida. (Morín, 1995, pp. 118-119)
} 
discurso sobre la mundialización ignora al mundo en sí mismo (...). La mundialización corresponde al surgimiento de problemas comunes y específicos para toda la humanidad. Pero la idea de humanidad es rechazada y considerada como obsoleta.

El carácter regresivo de la política económica global y local se instituye simultáneamente junto a múltiples procesos de complejización social originado por las transformaciones estructurales de la vida pública, privada, civil, política, cotidiana y subjetiva gestadas en los últimos dos lustros en Argentina y Latinoamérica.

La reducción y la negación de lógicas de organización social alternativas a las del modelo hegemónico refuerzan la idea de la insuperabilidad de la lógica de mercado pretendida como universalmente válido y extrapolable, como así también se pondera la "unicidad restrictiva" subyacentes en los postulados pragmáticos neoconservadores desde el cual se subsume el valor ontológico de la alteridad y la diversidad cultural real provocando una cosificación banalización de la diversidad transmutada en este contexto en comportamientos superfluos, estereotipados y "de baja intensidad" (Mc Laren, 1994), cuya validez depende exclusivamente de la abdicación del mercado de consumo.

Esta eliminación de la multiplicidad se llama (...) homogeneización (...) las expectativas y la peticiones, los deseos, las emociones y las gratificaciones, los juicios y las representaciones también imaginarias (...) todo esto se universaliza en firme y, por tanto, se resuelve en una igualdad que se enlaza como democrática, en verdad resultado e instrumento de una concertación autoritaria sin competencia, esto es monopolista. (Toesca, 1998, p. 359)

La incorporación de postulados "economicistas y pragmáticos", también en el campo de la gestión de las políticas educativas y sociales, propició la puesta en práctica de reduccionismos y disociaciones tecnocráticas avalada por principios racionalistas endilgados en los principios de modernización exigidos por las reformas estructurales que operaron como una deliberada y sistemática negación del antecedente histórico y social de la realidad educativa. Este hecho quedó plasmado en los objetivos y estilos de organización y planificación de los trabajos sociales de asistencia, ayuda y desarrollo social gestados desde el Estado y sus instituciones intermediarias durante los últimos 10 años. 
Desde estos múltiples condicionantes se dinamizan diversos procesos de exclusión enraizados "naturalmente" en la competencia neutral y radical de todos contra todos ${ }^{7}$ en consecuente desmedro de procesos sociales de inclusión sustentados por lo opuesto en valores como la solidaridad, la diversidad real y la cooperación, cánones que se volatilizan y erradican en un escenario social donde priman el egoísmo, la precariedad, el reciclaje e inseguridad inmanente como condiciones preponderantes de la organización social ${ }^{8}$; el ensimismamiento - autoreferencialidad, el individualismo negativo ${ }^{9}$ y el solipsismo de masas o muchedumbre solitaria.

En este especial contexto, histórico asistido por transformaciones sociales estructurales, nace el debate educativo con la obligación de volver a dirimir sus condiciones históricas, políticas y culturales, en torno a establecer una adecuada proyección de los marcos de referencia y argumentación del trabajo pedagógico (tanto en el ámbito formal como en el no formal) que pueda generar para los diversos grupos sociales, a saber:

Adaptaciones efectivas en la vida social, laboral y ciudadana.

> Participaciones ampliadas en todos los espacios institucionales de decisión y organización política y social.

> Estrategias de compensación, problematización (= desnaturalización) y superación de las desigualdades, marginalidades y "fronteras" que operan en y desde la escuela a través de los procesos de socialización (capitalización) de las herramientas cognitivas y culturales.

$>$ Una potenciación de las competencias, dominios e intereses individuales y colectivos de los sujetos aprendientes y de sus grupos de referencia.

\footnotetext{
7 La selectividad meritocrática empieza a tener implicaciones económicas, sociales y culturales. Competencia educativa significará ahora "¿ser competente para resolver tal cuestión o ser competitivo para ganar lugares excluyendo a otros?" (Cucuzza et al., 1996, p. 43)

${ }^{8}$ Al respecto, comenta Gorz (1998, pp. 221-223), los trabajos y las posiciones sociales son de por sí precarias. Raramente confieren un sentido de tener un puesto y una identidad seguros en la sociedad. Ni el nacimiento, ni la educación y ni si quiera el trabajo pueden asegurarlos ya. Los individuos están obligados a definir su propia identidad con sus propios medios, sin son capaces.

${ }_{9}^{9}$ Autores como Lechner, 1990, 1995; Bernstein, 1990; Beck, Giddens et al., 1997; Bourdieu, 1999; Beck 1990, 1998; Beck y Koch, 1999 se refieren a éste nuevo tipo de "individualismo" como un producto nocivo de las sociedades actuales, cuyos rasgos más distintivos son su no sociabilidad, vulnerabilidad, suceptibilidad, autoreclusión autoreferenciamiento, parcelamiento y dependencia de mandatos extrínsecos a sus capacidades psíquicas, sociales y afectivas para obrar de manera autónoma, crítica y desnaturalizante con el medio social y material de vida.
} 


\section{3) La incidencia de las transformaciones educativas en las transiciones de los jóvenes hacia el mundo del trabajo}

"La creencia que las buenas escuelas son el resultado de las buenas
elecciones es una concepción utópica porque cosifica la relación
entre la escuela y la sociedad y saca a la escuela de su contexto social". (Cookson, 1994, p. 101)

La presente crisis educativa se pliega a una crisis general que afecta a la sociedad en su conjunto y que se traduce en la acumulación de desventajas y aplazamientos en torno a la participación legítima a la educación, el trabajo, la vida digna, la seguridad social, etc. Este cuadro conflictivo se acentúa más entre los sectores populares (que históricamente se encuentran en situación de desventaja y vulnerabilidad) que registran en este período una endeble participación en las ofertas de formación educativa en las instancias tanto formal como no formal (CEPAL, 2000; 2001).

Si bien la matrícula en términos cuantitativos se incrementa notablemente durante el período 1989 - 1998 en el sistema educativo primario y los primeros años del secundario, el porcentaje de deserción, desgranamiento y repitencia se acrecienta paralelamente, fenómeno que permite constatar el carácter fuertemente heterogéneo, desigual e incongruente del servicio que reproduce el sistema educativo argentino (Sirvent, 1996).

Los efectos de esta crisis estructural determinaría que los sectores desfavorecidos -con inclusiones débiles e incompletas al sistema social- vean aún más reducidas y amenazadas sus escasas alternativas de apropiación objetiva de las herramientas sociales y cognitivas definidas también como capital cultural que se imparten en y desde la escuela y los centros de formación profesional y de educación no formal. A través de los efectos de la crisis se vigorizan las fronteras educativas en relación con el acceso de los jóvenes procedentes de sectores populares a los bienes del mercado de trabajo y consumo y se refuerza la vulnerabilidad de estos sujetos dotados de escasas alternativas educativas, fenómeno que se condice con una exclusión estructural que reproduce y anticipa históricamente las trayectorias de estos individuos y sectores sociales (Puiggrós, 1999).

En este contexto, los jóvenes "cercados" por limitaciones que restringen su acceso a los bienes y servicios sociales mínimos experimentan una doble marginación, a saber la primera, por ser "jóvenes", que desde la visión estigmatizada aún prevaleciente en la sociedad se sigue catalogando a la misma como sinónimo de falta de compromiso, transgresión, desinterés, 
pasividad, apatía, dejadez, superficialidad, hedonismo, etc. y, en segundo lugar, por ser "pobres", ya que se hallan circunscriptos por carencias, privaciones e insatisfacciones que determinan (desmedran) sus chances reales y potenciales de inclusión y permanencia en los diferentes ámbitos de la vida social, laboral, institucional, educativa, familiar, política, ciudadana, recreativa, asociativa, etc. (Margulis y Urresti, 1996; 1997).

La dinámica de reproducción de las condiciones de exclusión social, vale decir la permanencia de los excluidos y las polivalentes expresiones de las exclusiones, se cristalizan en la práctica social (explícita y subyacente) a través de negaciones y postergamientos a nivel político, institucional, económico y cultural, donde la "negación" y lo "negativo" como frontera estigmatizante descalifica en un continuum (Castel, 1995), cualquier instancia de reconocimiento, involucramiento o resistencia por parte de los sujetos que participan de una misma constelación histórica, política y social.

Ante el avance de una suerte de desmembramiento de los tejidos sociales, la institucionalidad de los axiomas organizadores de lo colectivo y la interpelación de los referenciamientos colectivos de la acción (Berger y Luckmann, 1995) la ruptura de "lo social" se traduce principalmente en una disociación progresiva y estigmatizada de los cuerpos, el debilitamiento de las solidaridades y la volatilidad de las instancias de participación, cohesión e identificación colectiva (Touraine, 1997).

Así pues, los fenómenos relacionados con la desafiliación, la individualización ${ }^{10}$ y la desposesión social (Bourdieu, 1997) que la política educativa actual pretende traducir como sinónimos de autonomía, responsabilidad personal, objetivación de las libertades locales e incremento del poder y responsabilidad de las coyunturas en la gestión del servicio educativo, etc., constituyen puntos neurálgicos del conflicto político reinante dentro del sistema educativo que inducen al desmoronamiento del potencial transformador que históricamente la sociedad y el Estado confirieron al rol de la política educativa como estrategia de desarrollo, espacio de cohesión y factor de movilidad social (UNESCO, 2001; 2001a; 2001b)

Con la introducción de estrategias reformistas llevadas adelante a través de los procesos de descentralización, privatización y "achicamiento" de la administración pública en el nombre

\footnotetext{
10 En términos de Lipoveztky (1986, pp. 10-22) este fenómeno se denominaría también como "proceso de personalización", a través del cual se habilita la neutralización y banalización de lo social, puesto que " (...) únicamente la esfera privada parece salir victoriosa de ese maremoto apático (...) en el que vivir sin ideales, sin objetivos trascendentes resulta posible" y desde donde además se tornaría plausible obtener de parte de estos sectores subsumidos en sí mismos una "aprobación muda, difusa, no política, del espacio democrático".
} 
de la "modernización y racionalización del aparato burocrático" se promovió una fragmentación anárquica del sistema educativo que afectó la totalidad de los recursos, actores y marcos de referencia profesional y laboral de los integrantes de la comunidad educativa (directivos, maestros, alumnos, planificadores, técnicos, especialistas, familias, etc.) y de los criterios políticos, administrativos, curriculares, didácticos, éticos, estéticos y epistemológicos que dieron sentido histórico a la necesidad de transformar la educación. De este modo, se propulsó un desgarramiento significativo del aporte transformador cultural de la escuela como institución socializada y formadora -en particular- y de la política educativa como espacio de decisión y proyección histórica y social -en general- (Hünermann et al., 2002).

Cabe señalar que la tradición prevaleciente en el sistema educativo previo a la intromisión de la reforma, esto es anterior a la implementación de la Ley Federal de Educación estaba ligada a un paradigma "burocrático centralista", esquema en donde el Estado de modo casi monópolico obraba como el principal responsable de la distribución, gestión y planificación de la oferta educativa; además estas funciones, eran desarrolladas con un vasto sesgo centralista, jerárquico, burocratizado y autoreferencial.

Empero a la intromisión propositiva de la transformación de la política educativa, se observa que el paradigma tradicional no fue sustituido del todo, y que sus efectos empíricamente evidenciables, se embretan fundamentalmente con la potenciación de la desigualdad socioeconómica, la marginación, la exclusión y la autoreclusión de los sectores sociales más desfavorecidos y entre ellos de los grupos más vulnerables, que en el caso de Argentina y los demás países de la región está entre los jóvenes sobre representados (Aparicio, 2005).

Estas dificultades constituyen, sin duda, las problemáticas pendientes más trascendentes por elucidar y dirimir, se puede constatar aún que no fueron asumidas a nivel político, burocrático y local con suficiente estructuralidad, previsión a largo plazo y cohesión intra, inter y transeducativa -en el sentido de consenso educativo amplio- que la naturaleza de dichas contradicciones lo exige.

\section{4) El desempleo entre las y los jóvenes, vulnerabilidad y desintegración social}

Entre una de las problemáticas más importantes que constriñe a la juventud argentina se destaca la desocupación y, en alusión a ella, la carencia de expectativas y confianza de los jóvenes en relación con el aporte real que suministran las instituciones de formación educativa 
en razón del incremento de sus chances personales y sociales (capital educativo acumulado), pues las mismas en la actualidad ya no aseguran ni la integración exitosa, armónica y a largo plazo ni el escalamiento seguro dentro de la estructura social.

Del mismo modo, la desorientación que suscitan las nuevas pautas organizadoras del mundo laboral, político, social y económico que han propulsado las tendencias a una mayor selección y exclusión social; fuerte versatilidad e inestabilidad laboral; gran velocidad en las instancias de transición y adaptación social exigidas por las nuevas estructuras del mundo económico, sumada a la ausencia de alternativas políticas en el espacio de juventud capaz de acompañar sistemáticamente la transición de estos actores, torna a este complejo panorama aún más confuso e inaprehensible para los jóvenes (Bourdieu, 1999; Medá, 1998; Galuske, 1998).

Los datos más recientes aseveran que la desocupación juvenil en Argentina alcanza los índices más altos de su historia $-46,6 \%$ para la cohorte de $15-25$ años- ${ }^{11}$, este hecho obliga a asentir como inobjetable que el deterioro, social, laboral y productivo en nuestro país acentúa la tendencia estructural hacia la restricción del empleo, la disminución (deterioro) de las oportunidades de integración laboral y la cuestionable efectividad de las propuestas políticas de formación educativa que se destinan a las nuevas generaciones, especialmente a los adolescentes y jóvenes, con el objetivo de inducir su integración social y económica en la vida adulta.

Actualmente, el Estado, la sociedad y el sector productivo, en el marco de la consecución de los procesos de modificación estructural del mercado de trabajo, enfrentan la difícil tarea de vislumbrar caminos intermedios que promuevan irrumpir sobre el "círculo vicioso" dado por la disociación entre: a) el cumplimiento de los requisitos de flexibilización, productividad $y$ eficiencia exigidos a las unidades de producción de servicios y bienes y el sector productivo local y global cual patrones macroeconómicos de gestión y administración desde donde se definen las estrategias de ampliación económica del capital y b) las consecuencias negativas y regresivas que se registran en los procesos de implementación de estas premisas en la realidad histórica social y en el sistema productivo y laboral que se instituye paulatinamente en la región y el mundo (OIT, 2000; Carlson, 2001).

La incertidumbre registrada en la organización, coordinación y planificación de políticas

\footnotetext{
${ }^{11}$ Ver Anexo Cuadro 1
} 
destinadas a la contención, apoyo y orientación laboral de los jóvenes responden, sin duda, a la remoción compleja que infringen los nuevos procesos de desplazamientos y reemplazamientos del mercado de trabajo, sus pautas de inclusión, sus disposiciones tecnocráticas, ideológicas y operativas.

Estos elementos coadyuvan a reforzar la volatilidad de los nexos integrativos e interactivos entre los dispositivos y los mecanismos político institucionales públicos, la iniciativa del sector privado y productivo y el conjunto de grupos y organizaciones provenientes de la sociedad civil. Todos estos actores hoy por hoy están inexorablemente implicados en las tareas de mejoramiento y potencialización social, político, jurídico y cultural de las condiciones de vida de los actores juveniles, entendidos éstos como un recurso estratégico del desarrollo integral de la sociedad que en Argentina y en América Latina ha venido asistiendo sistemáticamente al agravamiento de su situación de exclusión social y laboral (CINTERFOR/OIT, 2002).

El reclamo persistente a la política educativa por respuestas efectivas y relevantes se multiplican ante la incontención social que padecen los jóvenes en los diferentes ámbitos de la vida social, que sobredimensionan la impronta de la crisis laboral, económica y social dominante, para la cual cualquier tipo de respuesta remedial ya no puede erigirse de modo aislado, amparado por la dicotomización teórica que prioriza el valor y la lógica de lo económico en desmedro de lo pedagógico y viceversa.

Es por ello que uno de los grandes desafíos consiste en recuperar la impronta histórica e instituyente del discurso y la práctica educativa, que ante las conflictivas circunstancias históricas de la actualidad, sea capaz de replantear la instrumentación acrítica de criterios exclusivamente administrativos y gerenciales ligados más a la gestión, financiamiento, gerenciamiento estándar y estandarizante de sesgo economicista, pragmático, unidereccional y conductista, que prolongó la negación de las características históricas y sociales de los polivalentes constelaciones juveniles en cómo muchas de las nuevas propuestas de capacitación juvenil han sido introducidas (inyectadas) en los países de la región, bajo una tenue insinuación democrática y democratizante (Sirvent, 2000, 2001).

El desempleo juvenil se presenta como una amenaza y una obsesión omnipresente sostenida en un marco de condiciones estructurales y dinámicas históricas, sociales y políticas determinadas tanto a nivel local como global y que además, están fuertemente correlacionadas con determinadas estructuras de producción y de reproducción económica y social, modelos de trabajo y lógicas de distribución social del empleo. 
A continuación, y de forma breve, se enunciarán algunas problemáticas constreñidas al desempleo juvenil en Argentina, y entre las que cabrían destacarse:

* la incapacidad del mercado de trabajo para reclutar a grupos desprovistos de competencias, conocimientos y calificaciones actualizadas y suficientes; y garantizar a nivel universal una participación irrestricta en los servicios sociales tradicionalmente asociados a la posesión de empleos estables (seguridad social, ingreso mínimo, cobertura de salud, protección social y representación sindical, etc.) ${ }^{12}$ (OIT, 1998);

* el desajuste registrado entre las ofertas de formación profesional, técnica y académicas y las demandas de un mercado de trabajo asediado por serias limitaciones estructurales en lo referido a sus escasas estrategias de expansión, consolidación y sostenibilidad a largo plazo (Jacinto, 1996, 1997);

* la precarización de las oportunidades de empleo en términos cualitativos y cuantitativos que prevalecen en el actual mercado de trabajo; a ello se adhiere el incremento de la pobreza y la vulnerabilidad social que propulsa una tendencia a legitimar la subocupación (= depreciación y volatilización) de las competencias educativas y potencial de empleabilidad detentado por los jóvenes postulantes, tras la que se desemplean y volatilizan las competencias y dominios adquiridos en el sistema educativo en favor de maximizar los réditos de la producción, alivianar los costos del empleo y socavar la estabilidad del empleo y por ende las chances de vivir una cultura del empleo a costa de una permanencia transitoria subordinada a la voluntad de los empleadores y las "tendencias dominantes del mercado"; ${ }^{13}$

\footnotetext{
${ }^{12}$ Sobre la modificación del escenario laboral de los jóvenes Filmus et al. (2001, p. 99) exponen que "entre 1980 y 1999, entre quienes terminaron el secundario, el porcentaje de los que se encontraban en esa situación ascendió desde el $7,2 \%$ al $29,2 \%$. Si a esta tasa le sumamos los cuentapropistas y aquellos que se encuentran desocupados, es posible proponer que actualmente en la Argentina más de la mitad de los egresados de la escuela media no podrán acogerse al beneficio de la jubilación".

${ }^{13}$ Los jóvenes, debido a la necesidad imperiosa de generar ingresos económicos, son quienes comportan el mayor grado adaptabilidad y consentimiento ante los criterios de precarización, deterioro sistemático, aceptación pasiva y flexibilización que rigen y reseñan las tendencias del actual mercado de trabajo en Argentina (Monza, 1998). De este modo, se conforma un "círculo vicioso" dado por una introducción frágil y precaria en el sistema productivo, la congruente deficitaria formación educativa a la que se participa o se desiste ante los nuevos compromisos laborales asumidos y las escasas alternativas de inclusión social plena en el ámbito político, económico, civil y cultural". En consonancia con este diagnóstico y en alusión a la dinámica expansiva de los procesos de exclusión social Neffa et al. (1999:64) comentan que éstos afectaron "en forma heterogénea a los diferentes grupos sociales, siendo especialmente golpeados los más pobres, cuya tasa de desempleo de larga duración se multiplicó casi por 9 entre 1988 y 1997, subiendo de 0,6\% a 5,2\%. En cambio, en el estrato más alto, la tasa pertinente subió sólo de 0,2\% a $1,3 \%$ en las mismas fechas".
} 
* la desigual distribución social de las oportunidades educativas entre los jóvenes es congruente con el tipo diferenciado de acceso, la calidad del servicio educativo, la débil adecuación de las instituciones y los actores de formación, planificación y elaboración de políticas de capacitación, y cuya consecuencia primordial está dada por una inequitativa proyección de estos sujetos a nivel biográfico en el proceso de emplazamiento tanto en el mercado de trabajo como en la vida social. Es decir, en este contexto cundido por restricciones generalizadas lo que se torna un "privilegio de minorías" es la apropiación objetiva de la posibilidad de autorealización autónoma e integral;

* la prematura incorporación al mercado de trabajo de adolescentes y jóvenes vulnerables, que se ven obligados a contribuir al sustento económico de sus familias, culminaron abandonando total o parcialmente el sistema educativo, cuando estos sujetos prolongan el cumplimiento simultáneo de esta doble exigencia respectivas al espacio laboral y educativo. El bajo rendimiento educativo que se cristaliza en la noposesión de certificados o competencias suficientes y necesarias para participar en las diferentes órbitas de la vida social generan consigo una doble depreciación de los sujetos, a saber: a) depreciación de sí mismos, que acontece a nivel subjetivo y personal por no haber cumplido con las expectativas de las sociedades e individuales y b) depreciación practicada hacia ellos por parte del sector empleador, que se produce en un plano objetivo y estructural, manifestado cuando se deprecia el valor social del capital educativo detentado y por consiguiente se reducen las alternativas de mejoramiento y superación de las limitaciones que infringe un contexto cundido históricamente por "múltiples pobrezas" (Aparicio, 2003a; 2007). En el fondo, ambas manifestaciones del "desaliento" explicitan la tendencialmente baja plausibilidad de poder experimentar gracias al capital social y cultural detentado o por adquirir, un proceso de ascenso y realización social, profesional y/o laboral autónoma y plena en la estructura productiva;

* las demandas de trabajo con más relevancia se orientan a jóvenes con estudios medios completos e incompletos (alta tasa de escolaridad en comparación con las generaciones adultas de la PEA) que se desenvuelven flexible y pertinentemente frente a las nuevas tecnologías y dinámicas de trabajo. Así, el perfil del trabajador predominante recae sobre sujetos de menor edad, polivalentes, emprendedores, dotados de mayor formación educativa y que detentan un grado adecuado de preparación para asumir y 
acompañar el rigor de constantes reciclamientos de las diferentes unidades productivas y de servicios (Banco Interamericano de Desarrollo, 1998; Banco Mundial, 1995);

* la heterogeneización de las condiciones y posibilidades de empleo, subempleo ${ }^{14}$, sobreempleo, desempleo e inempleabilidad se expanden con notable fuerza en todos los sectores económicos y redimensionan las instancias, cláusulas y estructuras tradicionales ("normales") de producción, distribución y acumulación económica, y social conocidas en la sociedad (CEPAL, 2000aaㄱ;

* el paso de la formalidad a la informalidad ${ }^{15}$ cual desmedro sistemático de la calidad del empleo $^{16}$ y de la productividad a la improductividad (terciarización de la economía) respectiva a las actividades laborales deben ser interpretadas como las consecuencias más sobresalientes de los procesos de flexibilización implementados en el mercado de trabajo, que en Argentina evadieron "de facto" toda instancia real de consenso y acuerdo político multisectorial entre el gobierno, los sindicatos, la sociedad civil, etc. (Beccaria y López, 1996). Ambos fenómenos están constreñidos en la precarización del mercado de trabajo, que en toda la dimensión de la economía global afecta la distribución significativamente diferenciada del ingreso y de la riqueza entre los diferentes grupos sociales provistos de calificaciones, la asignación social de los puestos de trabajo y las oportunidades objetivas para desarrollar trayectorias laborales exitosas. En el marco de la flexibilización laboral se propulsaron la renovación de las modalidades de contratación

\footnotetext{
${ }^{14}$ El subempleo, en el mercado de trabajo argentino, está ligado al fenómeno de la "sobreeducación" de la fuerza de trabajo que desaprovecha a través del desempeño de tareas simples e improductivas. Esta tendencia se ha venido afianzando en el mercado de trabajo, que provoca, por ejemplo, que en la actualidad aproximadamente tres de cuatro jóvenes que obtuvieron empleo estén ocupados en tareas inferiores al grado de calificación poseído. Esto es lo que propulsaría sustancialmente el desaliento, la ausencia de expectativas y proyectos entre los jóvenes, en torno las pobres oportunidades de empleo que le aguardan -paradójicamente- a los sujetos que incluso detentan herramientas cognitivas y técnicas satisfactorias

${ }^{15}$ En apoyo a un estudio aportado por Filmus et al., (2001, p. 49) se puede aseverar que el sector informal de la economía continuó creciendo cual primordial incorporador y/o demandante de fuerza de trabajo. "La ocupación informal creció a una tasa anual superior a la del conjunto del empleo (3,9\%). Por eso, 6 de cada 10 nuevos empleos generados en la década del 90 fueron creados por este sector, lo que implicó que su participación en el total de los ocupados aumentara del $44,4 \%$ en 1990 al $47,9 \%$ en $1998 "$.

${ }_{16}$ Si consideramos que la estabilidad de los empleos se correlaciona con un mayor nivel de ingreso, óptimas condiciones contractuales, aceptables niveles de protección, un acceso más sólido y garantido a los servicios sociales básicos, es de notar que a partir de la mitad de los años 90 domina en el mercado de trabajo en Argentina una cruenta reducción a la creación de empleos catalogables como de buena calidad. "Solo 3 de cada 10 empleos creados en la década pueden incluirse en esta categoría; su participación en el total del empleo se reduce del 46,4\% en 1990 al 42,4\% en 1998." (Filmus et al., 2001, p. 51). Es de aquí que el notable crecimiento del sector informal produjo contiguamente a la disminución del sector moderno de la economía, que según consta en el mismo estudio mencionado disminuyó su participación en el empleo del 55,6\% en 1990 al 52,1\% en 1999.
} 
ahora más individualista y temporaria, así como la redeterminación de la carga horaria, las causas de despido y la asignación y cobertura social.

El desempleo en el contexto argentino y latinoamericano afecta principalmente a los grupos más expuestos y/o atravesados por la pobreza, la inseguridad, el riesgo, la precariedad y la desafiliación de la esfera política y social de vida. Vale decir, estos grupos "periféricos, externos y vulnerables" encarnan o reeditan en la actualidad al "ejército de reserva" distinguido por Marx (1994), cuya presión concomitante por el ingreso al sistema de producción provocaba y aún provoca la disminución de los salarios, la desintegración de los mecanismos sindicales de representación y consenso de intereses, y el desmoronamiento de los derechos sociales y laborales, con la gruesa diferencia de que la producción de ese momento con sus crisis de retracción productiva, en el momento de expansión "descansa en la constante formación, absorción más o menos intensa y reanimación del ejército industrial de reserva 0 superpoblación obrera" (Marx, 1994, p. 535).

Por el contrario, en la actualidad los ciclos de expansión se suplantan y se canalizan a través de innovación tecnológica, que producen una significativa diferenciación cuantitativa y cualitativa de los procesos productivos sin que esto tienda a mejorar y soslayar el destino de los excluidos, que inversamente proporcional a la modernización del empleo "ven progresivamente disminuidas sus posibilidades de reincorporación exitosa" (Bourdieu, 1999a , p.121)

\section{5) Avizorando referentes y oportunidades para una educación erigida desde y con los jóvenes}

El nuevo rol histórico, que deben asumir tanto la educación como la política educativa en relación al actor juvenil -dado el sostenido avance de los procesos de desintegración y exclusión social-, no puede definirse desde una visión estrictamente universal como pretende el discurso dominante de la globalización a través de postulados eficientistas y tecnocráticos, que pretenden seguir instituyéndose como únicos patrones de acción, programación y normalización válidos para la totalidad de los contextos sociales y culturales, que evidentemente no ponderan

\footnotetext{
17 La precariedad laboral afecta profundamente a quien la sufre; al convertir el futuro en algo incierto, impide cualquier previsión racional y, en especial, aquel mínimo de fe y esperanza en el futuro que es preciso poseer para rebelarse, sobre todo colectivamente, contra el presente, incluso el más intolerable.
} 
axiomas como la diversidad, heterogeneidad y localismos identificadores de los diversos grupos humanos.

A nuestro juicio, la nueva propuesta y orientación política educativa no puede ni debe ser el resultado exclusivo y parcial del ámbito pedagógico, donde intervengan solamente razonamientos didácticos, curriculares y epistemológicos, puesto que así se perpetúa la impenetrabilidad de lo "educativo escolarizante". La creciente complejidad de las nuevas formas de organización social producen incipientemente estilos y tejidos simbólicos y materiales que requieren ser incluidos y problematizados a nivel educativo, para así poder generar modalidades alternativas de formación y comunicación social capaces de integrar y recrear estas particulares formas de convivencia y transformación del mundo, tanto en el plano individual y colectivo como subjetivo y objetivo del comportamiento de los actores juveniles (Aparicio, 2003).

Consolidar un nuevo tejido de contención o red social de participación y formación de los jóvenes desde una perspectiva "pedagógica social", quizás sea el desafío más sobresaliente a desandar, desde donde se puedan generar estrategias congruentes y sistemáticas en interacción con otras organizaciones y sectores sociales para afrontar con mayor polivalencia problemáticas como la pobreza y los procesos de empobrecimiento, la desocupación y subocupación; la delincuencia juvenil; la desesperanza, soledad y desorientación de las nuevas generaciones frente a un mundo atravesado por la incertidumbre, la insolidaridad y una competencia letal de "todos contra todos" pretendida por el mercado, fenómenos que impactan especialmente sobre los estilos de convivencia, participación e integración social.

A través de la construcción democrática de una oferta educativa ampliada e implicante del complejo espacio social e histórico de los jóvenes desde la Pedagogía Social se pretende consolidar los valores ligados a la cohesión social, la gobernabilidad democrática y el desarrollo de nuevas formas de organización política y social capaz de integrar diferentes sectores y actores sociales, en una pluralidad real que habilite la discusión, localización y complementariedad significativa entre la oferta del servicio educativo y el universo social a donde dichos postulados se orientan y significan (Thiersch, 1984; 1998).

La asunción política de la diversidad de las problemáticas y contradicciones sociales -que asiste particularmente al universo juvenil- permitirá organizar un sistema educativo flexible, que resulte una herramienta idónea para el diagnóstico histórico situacional de los actores y/o grupo social de referencia (Hünermann y Eckholt, 1998). Disponiendo de una adecuada gestión y 
coordinación de alternativas políticas desde la educación que van "más allá de la escuela" se puede incorporar a múltiples instituciones sociales como la iglesia, comunidades de base, ONGs, centros vecinales, sindicatos, clubes, ligas de familias, centros y dispensarios de ayuda social, ligas de familia, entidades públicas y recreativas, etc., en miras a mejorar y diversificar las oportunidades y condiciones de participación efectiva en el ámbito educativo, social, civil y laboral de los jóvenes (Álvarez y Aparicio, 2003).

De este modo, se tornaría plausible integrar democrática, educativa y socialmente no solo al sujeto social desde una visualización pedagógica como "sujeto del aprendizaje", sino mucho más, como "sujeto de la complejidad" y de su propia complejidad. Es decir, dicho sujeto, desde la visión pedagógica social, debería ser asumido como "un proceso abierto" (Morín), capaz de reconocerse y de ser reconocido histórica y dialécticamente como un componente implicado e implicante, sujeto construido y co-constructor de las condiciones sociales, políticas, materiales y culturales específicas que involucran su existencia (Freire, 1992; 1997).

El sistema educativo argentino constreñido aún en una tradición centralista, autoreferencial y burocrática debe adquirir un nuevo reto y compromiso consistente en potenciar el ingreso y paulatino protagonismo de diversos actores y contextos sociales vinculados con la educación. Para ello, es primordial coordinar estrategias políticas de decisión, planificación y evaluación participativa en el servicio educativo, que alteren los circuitos y mecanismos de reproducción de las pobrezas, deserciones y desgranamientos explícitos e implícitos, así como reales y potenciales, que atraviesan a la actual oferta pedagógica (Aparicio, 2008).

La aparente igualdad que plantea la educación pública, gratuita y obligatoria, y que estaba estipulada en el corpus de la Ley Federal de Educación, subsumía y negaba la preponderancia de un problema estructural ligado a la desiguladad y su pontencial consecuencia: la diferenciación social, que nacida desde la entraña misma del sistema educativo desmedró la sostenibilidad de un discurso transformador y comprometido con las deudas pendientes de antaño.

La escasa búsqueda de estrategias y mecanismos políticos de compensación contextualidos y empoderizantes, vale decir que hicieran eje en las causas de la desigualdad y de las brechas sociales y educativas prevelentes acabó por instalar el modelo de una sociedad dividida en clases, sectores y fronteras.

La desigualdad educativa amparada y potenciada en la desigualdad social irrumpieron como elementos funcionales del círculo vicioso de la exclusión, que bajo el amparo de la 
hegemonía neoliberal derivó en una mayor indefensión y segregación de los grupos desaventajados (Puiggrós, 1994).

En este contexto, la ampliación de los criterios que estiman la dimensión de las fronteras, los riesgos y la desigualdad instaladas en el seno de la escuela -en particular- y del sistema educativo -en general- se presenta como una demanda urgente a soslayar, sobre todo, en el marco de una coyuntura donde la creencia en la legitimidad de la educación como estrategia política y cultural de transformación social y la escuela como institución educativa de promoción y formación sociocultural atraviesan una crisis de desprestigio político y social históricamente exponenciales (Popkewitz, 1998).

Bosquejar una estrategia sistemática y eficiente que pueda atemperar la dualización planteada entre los valores democráticos de ciudadanía, integración y participación social frente a los ápices de exclusión elusión de las contradicciones inherentes en el presente actual sistema educativo y la güetización de lo social que se experimentan en nuestros días, así como también entre erosión y marginación social, educativa y económica de los sectores sociales respaldan los claros argumentos que apoyan la tesis de bregar por una reforma educativa sistemática, que esté constreñida histórica y dialécticamente en la vida cotidiana, en los hábitos, en el sentido común, en las cosmovisiones y las representaciones y así también en el marco de condiciones estructurales que atañen la vida de los actores, las instituciones educativas y la comunidad. $^{18}$

La promoción de nuevas investigaciones en el ámbito de juventud, la creación de espacios concretos y reconocidos destinados a la participación y discusión juvenil y la viabilización de políticas de juventud formuladas por el Estado en coordinación con el resto de las políticas públicas y privadas dispuestas sistemáticamente entre diversas instituciones involucradas e interesadas en la formación educativa de los jóvenes representan algunos de los eventuales cimientos sobre los cuales se tendría que edificar el nuevo escenario político de la juventud.

En este sentido, la acción educativa de capacitación juvenil debe concebirse como herramienta compleja de síntesis, innovación y reconocimiento de las demandas, necesidades y

\footnotetext{
18 En las fronteras de la exclusión las escuelas resemantizan la articulación entre educación y pobreza quedando situadas en un campo de posiciones en que se balancean entre la posibilidad de fortalecer los vínculos de inclusión y la reproducción y profundización de la exclusión. Esta tensión cobra significados en la cotidianeidad escolar". Redondo y Thisted 1999, en Puiggrós et al., (edit.), p. 157
} 
exigencias de este grupo. Ella debería además de gestarse sobre una plataforma epistemológica, práctica y política ecléctica, donde pueden converger miradas y dispositivos polivalentes y polisémicos, procedentes de diferentes disciplinas científicas, organizaciones públicas y privadas y demás asociaciones civiles.

Rebasar la formalidad y universalidad en cómo se presuponen las necesidades existenciales del mundo de la vida de los jóvenes (Lebenswelt) se erige en este contexto específico como una condición sine qua non para poder empezar a reconocer a los actores no sólo como clientes de políticas, beneficiarios de proyectos de asistencia y ayuda, o bien meros objetivos referenciales de acciones exógenas, sino más bien como autores y actores de políticas, como referencias vivientes, críticas, dialógicas y dialécticas de las políticas y los proyectos de transformación social en el área de formación educativa (Aparicio y Carbajal, 2009).

La pronunciada asimetría planteada entre los objetivos sociales del Estado y la mirada que determina la agenda política donde yacen expuestas las prioridades más urgentes, se sostiene en la auto-convicción -desde el discurso político oficial- de que el fracaso de todas las propuestas de intervención laboral, social y educativa en el ámbito juvenil, en el fondo, se deben a resistencias anómicas, "rechazos tercos" y auto-negación voluntaria de los sectores marginales y excluidos en el ámbito de la formación, la asistencia social y laboral. Esto nos motiva a aseverar que el modo en cómo se extiende un servicio o programa no se puede disociar de los cánones en cómo el grupo objetivo se asume histórica y socialmente.

En consecuencia, las herramientas metodológicas, teóricas, técnicas, didácticas y pedagógicas empleadas en todo proceso de formación deben favorecer una mayor visibilización y vinculación del conjunto de demandas, expectativas y potencialidades de las personas, solo así resulta pensable una transformación genuina del espacio educativo de formación.

En esta directriz el mayor reto estriba en reemplazar la reforma educativa planteada en una lógica de asistencia o inyección "bancaria" (Freire, 1992), enajenante y unidireccional de modelos teóricos y dispositivos curriculares que ha sido el sustrato de muchos proyectos de formación y empleo juvenil llevados a cabo en Argentina. El resultado de esta lógica asimétrica y jerárquica de diseñar y gestionar políticas en el ámbito de juventud ha propendido a exacerbar la disposición clientelar y pasiva de los actores educativos, desmedrando el contenido democrático y empoderizante de los programas y una genuina mejora de las oportunidades de participación y desarrollo cognitivo, social y laboral. 
Es por ello que el consenso social multisectorial, entre diversos actores sociales, constituye una premisa trascendente en el ámbito de la política educativa, social y laboral destinadas a los jóvenes que compromete no sólo el éxito y la relevancia de los programas, los objetivos y el poder de impacto de la inversión social en torno al contrarrestamiento de problemáticas como la pobreza y la desocupación, sino que principalmente afecta el grado de recepción, apropiación y capitalización que la sociedad en su conjunto y especialmente el grupo objetivo elaboran a nivel social e histórico.

Generar espacios y herramientas de contención e integración social que no se diluyan en la univocidad unilateralidad laboral, productiva $u$ ocupacional, bajo los términos que el capitalismo define como trabajo productivo y sus correspondientes relaciones de beneficio y reproducción social y material, exigen sin duda la necesidad de pensar nuevas formas de redistribuir entre muchos sectores desplazados -que en su mayoría son las más pobres, vulnerables, descalificados, excluidos, desprotegidos y desafiliados- el privilegio de participar parcial o plenamente en el mercado de trabajo fuertemente selectivo e inapelable en razón a los "grupos perdedores", intentando establecer un equilibrio democrático, equitativo y solidario (garantido por el Estado en consonancia con el mercado y la sociedad civil) entre los diversos sectores sociales, sin descuidar los valores de la eficiencia, la competencia y la realización personal de las personas en el acto de producción, decisivos para poder permanecer en el circuito global y local económico.

Para concretizar estos fines es imprescindible la asunción de un rol comprometido y estratégico por parte del Estado, cuya intervención se torna determinante para la constitución de consensos multisectoriales y la construcción de nuevos espacios de formación y acción juvenil desde donde a su vez sea posible:

$>$ efectivizar una socialización adecuada de competencias, saberes, disposiciones y valores relevantes en términos subjetivos y sociales;

$>$ contribuir a la readaptación de las instituciones de formación que permita articular de modo efectivo las "propuestas marco" -ligadas a la centralización normativa- con los múltiples contextos y referencias locales "micro" propulsados por una descentralización ejecutiva; y

> construir herramientas políticas flexibles y estrategias focales basadas principalmente en el criterio de "discriminación positiva" (Gallart) que no debe confundirse con la perpetuación de propuestas atomísticas (sin posibilidad de integración en una 
estructura política de mayor cobertura y envergadura), residuales (desprovistas de toda prioridad y valoración política) u obsoletas (desposeídas de actualización y trascendencia histórica social).

La construcción de esta nueva forma de pensar, asumir y actuar educativamente con y desde los jóvenes -creemos- se presenta como un eslabón determinante para la confección de nuevas ofertas de integración, organización y participación juvenil.

Posibilitar el reconocimiento subjetivo de los actores a través de la viabilización política e institucional de un proyecto de formación socio educativo integral y próximo a los intereses de los jóvenes y sus referentes colectivos y locales constituye una apuesta decisiva para poder superar el atisbo de "la frustración" y avanzar hacia "un cambio real" de las dinámicas productivas y reproductivas de restricciones prevalecientes en la actualidad dentro del ámbito laboral, social, económico y educativo.

\section{Referencias}

Álvarez, Claudia y Aparicio, Pablo Christian. (2003). Öffnung der Schule in Argentinien und Chile aus der Perspektive der Jugendhilfe, Recuperado el 12 de marzo de 2011, de: http://tobias-lib.uni-tuebingen.de/frontdoor.php?source opus=873\&la=de Biblioteca de la Eberhard-Karls Universität Tübingen - Alemania

Aparicio, Pablo Christian. (2003). Die Bedeutung des kulturellen Kapitals für die Sozialisationsprozesse der argentinischen Jugendlichen im Bildungssystem: Zugang zur Demokratisierung oder sozialer Ausschluss? Risiko oder Chance? Recuperado el 14 de marzo de 2011, de: http://tobias-lib.unituebingen.de/frontdoor.php?source opus=872\&la=de Biblioteca de la Eberhard-Karls Universität Tübingen - Alemania

Aparicio, Pablo Christian. (2003a). La pertinencia de las nuevas políticas educativas implementadas en un contexto de "multiples pobrezas". Revista Temas Sociológicos (9), 161-200. Universidad Católica Raúl Silva Enríquez.

Aparicio, Pablo Christian. (2004). Breve reseña del actual escenario educativo argentino y su posicionamientofrente a la dilemática articulación entre trabajo, formación educativa y juventud. Revista Iberoamericana de Educación, 39 (19), 1-13. Recuperado el 4 de marzo de 2011, de http://www.rieoei.org/pol edu6.htm

Aparicio, Pablo Christian. (2005). Los jóvenes, educación y política de juventud en Argentina. Restricciones y desafíos de la integración social en el contexto de la globalización. Educere, Año IX (30), 287-269. Mérida, Venezuela: Universidad de Los Andes. 
Aparicio, Pablo Christian. (2007). Los jóvenes y la vertiginosa transformación educativa, social y laboral. Una aproximación socio educativa a la realidad a la realidad de los/as jóvenes argentinas/os. Studi sulla Formazione, Año IX (2), 86-106. Florencia: Gedit Edizione, Universidad de Florencia.

Aparicio, Pablo Christian. (2008). Los difíciles caminos hacia la integración educativa y laboral de los jóvenes en América Latina. EDUCERE. Revista Venezolana de Educación, Año 12 (43), 719-729. Recuperado el 4 de marzo de 2011, de http://www.saber.ula.ve/handle/123456789/26695

Aparicio, Pablo Christian. (2008a). The Young People and the Challenges of Educational and Work Inclusion in Argentina after the Structural Changes of the 90's. Causes, Dynamics and Consequences. Revista Electrónica de Investigación Educativa, 10 (1), 1-22, Baja California. Recuperado el 8 de marzo de 2011, de http://redie.uabc.mx/vol10no1/contentsaparicio.html

Aparicio, Pablo Christian y Carbajal, Sergio. (2009). Construyendo puentes de integración entre la educación y el trabajo. Perspectivas de empleo y los procesos de participación en Argentina. Revista Temas Sociológicos, (13), 109-139. Universidad Católica Raúl Silva Enríquez.

Banco Mundial (BM). (1995). El mundo del trabajo en una economía integrada. Washington: BM

Banco Interamericano de Desarrollo (BID). (1998). América Latina frente a la desigualdad. Progreso Económico y Social en América Latina. Informe 1998-1999, Washington: BID

Beccaria, Luis \& López, Néstor. (Comp.)(1996). Sin Trabajo. Buenos Aires: UNICEF/ Losada

Beck, Peter y Koch, Josef. (Edit.) (1999). Was ist normal? Normalitätskonstruktionen in Jugendhilfe und Jugendpsychiatrie, Weinheim und München: Juventa

Beck, Ulrich. (1990). Risikogesellschaft. Auf dem Weg in eine andere Moderne. FrankfurtMain: Suhrkamp

Beck, Ulrich. (1998). ¿Qué es la globalización? Falacias del globalismo, respuestas a la globalización. Barcelona: Editorial Paidós

Beck, Ulrich, Giddens, Anthony et al. (1997). Reflexive Modernisierung. Frankfurt a. M.: Suhrkamp

Berger, Peter L. y Luckmamm, Thomas. (1995). La construcción social de la realidad. Buenos Aires: Amarrortu Editores

Bernstein, Basil. (1990). Poder, educación y conciencia. Barcelona: El Roure Ed.

Bourdieu, Pierre. (1997). Capital cultural, escuela y espacio social. Buenos Aires: Siglo XXI Editores 
Bourdieu, Pierre. (1999). Meditaciones pascalianas. Barcelona: Editorial Anagrama

Bourdieu, Pierre. (1999a). Las miserias del mundo. Madrid: Akal

Carlson, Beverley. (2001). Education and the labour market in Latin America: Why measurement is important and what it tells us about policies, reforms and performance. Santiago de Chile: CEPAL

Castel, Robert. (1995). Les métamorphoses de la questione sociale. Une chronique du salariat. París. Arthéme Fayard

CEPAL (Comisión Económica para América Latina y el Caribe). (2000). Equidad, desarrollo y ciudadanía en América Latina. Santiago de Chile: CEPAL

CEPAL (Comisión Económica para América Latina y el Caribe). (2000a). Juventud, población y desarrollo en América Latina y el Caribe. Síntesis y conclusiones. Santiago de Chile: CEPAL

CEPAL (Comisión Económica para América Latina y el Caribe). (2001). Protagonismo juvenil en proyectos locales. Lecciones del Cono Sur. Santiago de Chile: CEPAL

CINTERFOR- OIT (Centro Interamericano de Investigación y Documentación sobre Formación Profesional - Organización Internacional del Trabajo). (2002). Documento del Seminario Virtual I Juventud y formación para la empleabildad: desarrollo de competencias laborales claves. Montevideo: CINTERFOR-OIT

Cookson, Meter. (1994). Schools choice. The struggle for the soul of american education. Binghamton: NY Yale University

Cucuzza, Roberto et al. (1996). Historia de la Educación en debate. Buenos Aires: Miño y Dávila

Filmus, Daniel. (1998). Hacia una nueva articulación en la relación educación - trabajo. En Daniel Filmus (comp.) Los noventa. Política, sociedad y cultura en América Latina y Argentina de fin de siglo (pp.193-220). Buenos Aires: Eudeba/FLACSO

Filmus, Daniel. (1999). Estado, sociedad y educación en la Argentina de fin de siglo, Buenos Aires: Troquel

Filmus, Daniel. (2002). La educación media latinoamericana frente a la crisis del mercado de trabajo. En P. Hünermann; V. P. Valera y H. Neuser (edts.), Formar, educar y aprender (pp. 201-216). Buenos Aires: Temas

Filmus, Daniel et al. (2001). Cada de más, necesaria, cada vez más insuficiente. Escuela media y mercado de trabajo en épocas de globalización. Buenos Aires: Santillana

Fitoussi, Jean - Paul \& Rosenvallon, Pierre. (1997). La nueva era de las desigualdades. Buenos Aires: Editorial Manantial 
Freire, Paulo. (1990). La naturaleza política de la educación. Barcelona: Siglo XXI Editores

Freire, Paulo. (1992). La pedagogía del oprimido. La Paz: Inti

Freire, Paulo. (1997). Política y Educación. Madrid: Siglo XXI Editores

Friedman, Milton y Rose. (1993): Libertad de elegir. Barcelona: Editorial Grijalbo

Galuske, Michael. (1998). Jugend ohne Arbeit. Das Dilemma der Jugendberufhilfe. Zeitschrift für Erziehungswissenschaft, 1, 535-560

Gorz, André. (1998). Métamorphoses du travail. Quête du sens. Critique de la raison économique. Paris: Galilée

Gorz, André. (2000). Arbeit zwischen Misere und Utopie. München: Suhrkamp

Hünermann, Peter y Eckholt, Margit (edit.). (1998). La juventud latinoamericana en los procesos de globalización. Una opción por los jóvenes. Buenos Aires: Eudeba / Flacso

Hünermann, Peter; Pérez Varela, Víctor \& Neuser, Heinz. (edit.). (2002). Formar, educar y aprender. Buenos Aires: Temas

Jacinto, Claudia. (1996). Desempleo y transición educación - trabajo en jóvenes de bajos niveles educativos. De la problemática estructural a la construcción de trayectorias, Revista Dialógica. Buenos Aires: CEIL, pp. 65-81

Jacinto, Claudia. (1997). Políticas públicas de capacitación laboral de jóvenes. Un análisis desde la expectativas y las estrategias de los actores. Boletín Técnico Interamericano de Formación Profesional, Boletín CINTERFOR/OIT N¹39-140, Motevideo: CINTERFOR/OIT pp. 57-88

Jameson, Fredric. (1996). Teoría de la postmodernidad. Madrid: Editorial Trotta

Lechner, Norbert. (1982). El proyecto Neoconservador y la Democracia. Revista Crítica y Utopía, (6), 39-77, Buenos Aires

Lechner, Norbert. (1995). Debate sobre estado y mercado. Revista Nueva Sociedad 121, 2542. Caracas

Lechner, Norbert. (1999). Los condicionantes de la gobernabilidad democrática en América Latina de fin de siglo. En Daniel Filmus (comp.) Los noventa. Política, sociedad y cultura en América Latina y Argentina de fin de siglo (pp.76 -80). Buenos Aires: FLACSO

Lipovetsky, Gilles. (1986). La era del vacío. Barcelona: Editorial Amagrama 
Mancebo, Martha. (1998). La sociedad Argentina de los '90: crisis de socialización. En Daniel Filmus (comp.). Los noventa. Política, sociedad y cultura en América Latina y Argentina de fin de siglo. Buenos Aires: Eudeba/FLACSO, pp. 177- 200

Margulis, Mario y Urresti, Marcelo (eds.). (1996). La juventud es más que una palabra. Buenos Aires: Biblos

Margulis, Mario y Urresti, Marcelo (eds.). (1997). La cultura en la Argentina de fin de siglo. Ensayos sobre la dimensión cultural. Buenos Aires: Oficina de Publicaciones del CBC, UBA

Marx, Kart. (1994). El Capital. Buenos Aires: Fonde de Cutura Económica

Mc Laren, Meter. (1994). Pedagogía crítica, resistencia cultural y producción del deseo. Buenos Aires: REI Argentina - Aique Grupo Editor S.A.

Méda, Dominique. (1998). El trabajo. Un valor en peligro de extinción. Barcelona: Gedisa

Monza, Alfredo (1998). La crisis del empleo en la Argentina de los 90. En Aldo Isuani y Daniel Filmus (comp.), La Argentina que viene. Análisis y propuestas para una sociedad en transición (pp. 223 - 261). Buenos Aires: Grupo Editorial Norma

Morín, Edgar. (1995). Introducción al pensamiento complejo. Barcelona: Editorial Gedisa

Morín, Edgar \& Nair, Samin. (1997). Une politique de Civilización. París: Arlea

Neffa, Julio; Battistini, Osvaldo; Panigo, Demian \& Pérez, Pablo. (1999). Exclusión social en el Mercado del trabajo. El caso de Argentina. Santiago de Chile: Oficina Internacional del Trabajo y Fundación Ford

OIT (Organización Internacional del Trabajo). (1998). Informe América Latina y el Caribe. En Panorama Laboral '98, (5). Lima: OIT

OIT (Organización Internacional del Trabajo). (2000). América Latina y el Caribe. Panorama Laboral 2000. Lima: OIT

Popkewitz, Thomas. (1998). Los discursos redentores de las Ciencias de la Educación. Sevilla: Ediciones Morón

Puiggrós, Adriana. (1994). Imaginación y Crisis en la Educación Latinoamericana. Buenos Aires: Alianza Editorial

Puiggrós, Adriana et al. (Comp.)(1999). En los límites de la educación: niños y jóvenes del fin de siglo. Buenos Aires: Homo Sapiens.

Redondo, Patricia y Thisted, Sofía. (1999). Las escuelas primarias 'en los márgenes'. Realidades y futuro. En Adriana Puiggrós et al (edit.), En los límites de la educación: niños y jóvenes del fin de siglo. Buenos Aires: Homo Sapiens. 
Sirvent, María Teresa. (1996). La educación de jóvenes y adultos en un contexto de políticas de ajuste, neoconservadurismo y pobreza. Revista del Instituto de Investigaciones en Ciencias de la Educación, Año V, (9), 65-72. Universidad de Buenos Aires - Miño y Dávila Ed.

Sirvent, María Teresa. (1999). Cultura popular y participación social. Madrid/Buenos Aires: Miño y Dávila

Sirvent, María Teresa. (2000). La función política y social de la educación. Los valores frente al neoliberalismo. Voces, Año IV, (7), 42-49 Uruguay

Thiersch, Hans. (1984). Sozialpädagogische Handlungskompetenz, die Frage nach der Tradition pädagogischen Handelns und das Konzept der Krisenidentität. En Müller, Siegfried et al (Edit.), Handlungskompetenz in der Sozialarbeit/Sozialpädagogik. Bielefeld

Thiersch, Hans. (1998). Notizen zum Zusammenhang von Lebenswelt, Flexibilität und flexiblen Hilfen. En Peters/ Trede/ Winkler (Hg) Integrierte Erziehungshilfen. Qualifizierung der Jugendhilfe durch Flexibilisierung und Integration (p. 301). Frankfurt/Main

Tenti Fanfani, Emilio. (1996). Cuestiones de exclusión social y política. En Alberto Minujin (editor), Cuesta Abajo. Los nuevos pobres: efectos de la crisis en la sociedad argentina. Buenos Aires UNICEF- Losada, pp. 241-274

Tenti Fanfani, Emilio (Comp.). (2000). Una escuela para los adolescentes. Reflexiones y valoraciones. Buenos Aires: UNICEF / Editorial Losada

Toesca, Paolo. (1998). Tiempos modernos. En García Wiedermann, E. (edit.), Los tiempos d la libertad (pp. 359 - 372). Buenos Aires: Ediciones del Serbal

Touraine, Alain. (1997). ¿Podremos vivir juntos? Brasil: Fondo de Cultura Económica

UNESCO. (2000). Marco de Acción Regional de la Educación de Personas Jóvenes y Adultas (EPJA) en América Latina y el Caribe (2000 - 2010). Santiago de Chile: UNESCO/OREALC

UNESCO. (2001). Análisis de Prospectivas de la Educación en América Latina y el Caribe. Santiago de Chile: UNESCO/OREALC

UNESCO. (2001a). Balance de los 20 años del Proyecto Principal de Educación en América Latina y el Caribe. Santiago de Chile: UNESCO/OREALC

UNESCO. (2001b). El futuro de la educación en América Latina y el Caribe. Santiago de Chile: UNESCO/OREALC 


\section{Anexo 1}

\section{América Latina: Tasa Desempleo Juvenil Primer Trimestre del 1998 a Primer Trimestre del 2002 (Datos en porcentajes)}

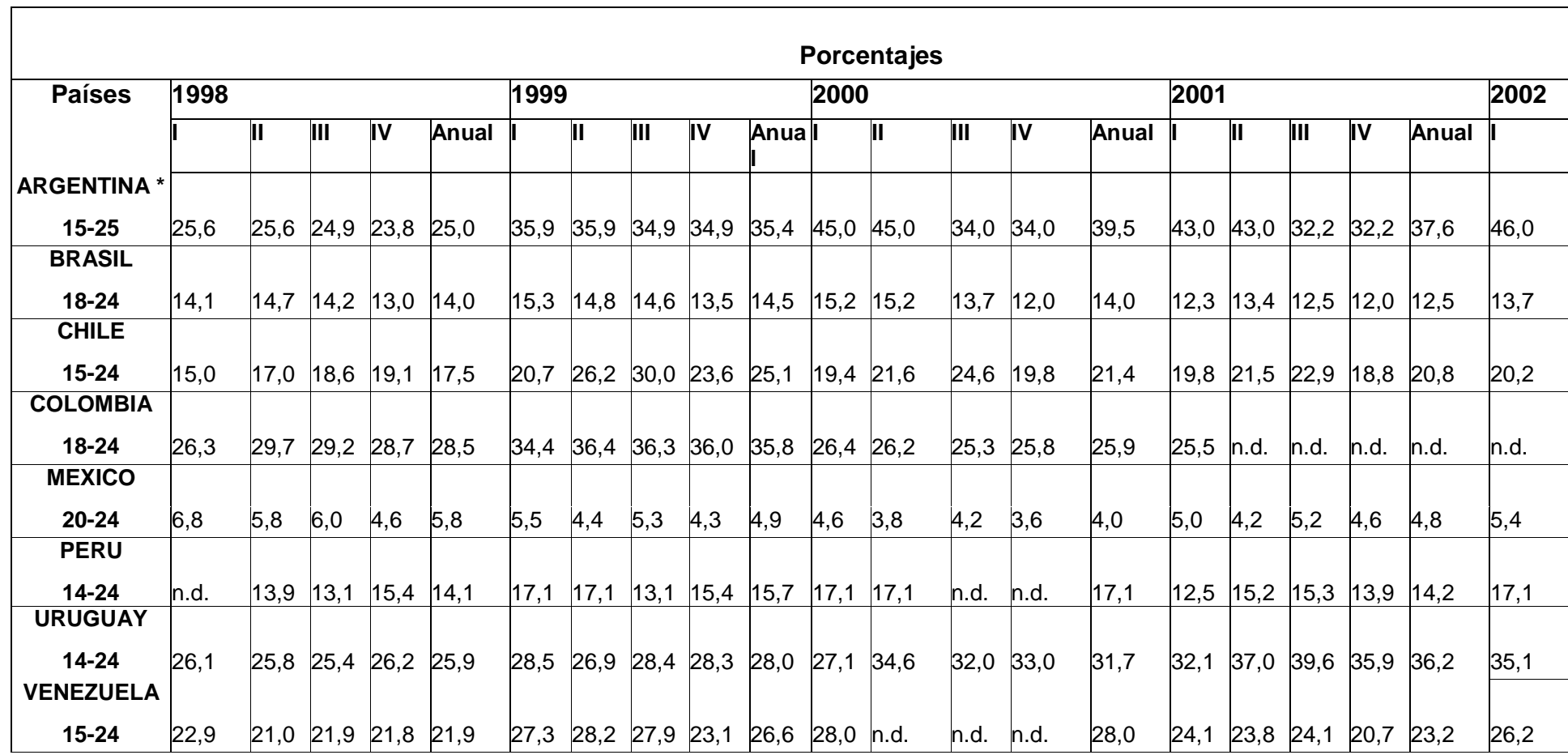

Fuente: Elaboración OIT, con base en datos oficiales de los países. Material del Seminario Virtual I Juventud y Formación para la empleabilidad: desarrollo de competencias laborales claves. Montevideo, 28 de Octubre de 2002. Centro Interamericano de Investigación y Documentación sobre Formación Profesional (CINTERFOR) dependiente de la OIT e Instituto de la Juventud del Ministerio de Trabajo y Asuntos Sociales (INJUVE/MTAS - España), p.5. Ver: http://www.cinterfor.org.uy/seminariojovenes Notas: (*) Gran Buenos Aires. A partir de 1999 se considera el segmento de 15 a 19 años. 